\title{
PUTIAM'S COMPLAINT
}

\author{
Jack K. Horne $\mathrm{r}$
}

One of the most acrimoniously disputed issues in contemporary philosophy of language is whether or not, or in what sense, humans may be said to possess an innate language-specific mobilization and acquisition capacity. At the heart of this dispute lies a slogan proponents of linguistic innatism have proclaimed as their credo of war, the so-called "innatist hypothesis" (I.H., for short): the human brain is "prograinined" at birth with some quite specific and structured aspects of human natural language; in particular, every linguistically competent human innately possesses a class $S$ of trans formational grammars $G_{1}, G_{2}, \ldots$, and a function $f$ which assigns weights to elements of this class in a manner modulated by experience. All members of $S$ are held to share certain very general features known as "linguistic universals" such as an activepassive distinction, a transformational component, linguistic categories such as concrete noun, verb taking an abstract subject, and so on. This hypothesis, the proponents of linguistic innatism claim, is virtually unavoidable on any account of linguistic behavior which adequately accounts for both the existence of linguistic universals and the empirical facts of language acquisition.

Several philosophers have balked at accepting the I.H.; indeed, some have found the proposal strong evidence of its proponents' senility.1 Prominent among the movements of this antagonistic requiem is the ubiquitous recitative of Professor Hilary Putnam.2 Yet Putnam's lamentation is, if I an not mistaken, a prelude to a premature burial. For if $I$ understand him correctly, Professor Putnan's arguments do not, for a variety of reasons, succeed in impugning the innatist view. To elaborate this criticism, I will first briefly review several linguistic phenomena which I hold an adequate theory of linguistic behavior must explain; Putnam's remarks will then be assessed against these data.

The first datum which an adequate theory of language must be able to explain is what I will call the "creative" aspect of language. Much of what we say or recognize as well-formed parts of language is new to us, in the sense that it is not merely a repetition of anything we have heard before, nor is it, in any important sense, a repetition of patterns we 
have experienced before.

Secondly, it can be shown that the number of sentences which a normal adult user of language can potentially recognize as grammatical is infinite; certain subclasses of English, for example, have been shown to have infinite cardinality. Accordingly, an adequate linguistic theory must account for such a capacity, given the temporally limited resources of the organism. There is only one way that such a capacity could exist, namely, if the structure of language acquisition or recognition could in effect be characterized by a set of recursive rules capable of generating the set of well-formed expressions of the natural language. 3

Thirdly, it is a reasonably well-accepted observation that children acquire a grammar based on very degenerate evidence or reinforcement. For example, many of the expressions they encounter during the language acquisition period are not well-formed: adult speech often contains slurs, false stops, spurious phonemes, and so on. Yet these errors are rejected as such very early in the child's linguistic development. More generally, the linguistic "input" which children receive by the time they may be said to possess linguistic competence does not determine the grammar they actually acquire. This fact can be shown without appealing to elaborate evidence, in the following way. At any given time $t_{0}$ in a child's life, he will have experienced a finite set $K_{0}$ of distinct well-formed expressions $E_{1}, \ldots, E_{p}$ of hiS language. Consider, then, the following vely crude grammars:

(RI) Only elements of $K_{0}$ are well-formed
expressions.

(R2) Let $K$ be the class consisting of the following

(i) $E_{j}, \ldots, E_{\mathbb{L}} \in \mathrm{K}$;

(ii) any conjunction of members of $K$ is a member of $\mathrm{K}$

Any meinber of $K$ is well-formed.

Note first of all that any expression satisfying $R I$ satisfies R2, but not conversely. This implies that the elements of $K_{0}$ satisfy both $R 1$ and $R 2$. Hence $K_{0}$ moreover, the list of rules above can be extended indefinitely. "Accordingly, any given finite set of expressions satisfies an infinite number of grammatical rules, and thus $K_{0}$ is not sufficient to determine even a finite class of ${ }^{0}$ rammars. Hence an adequate theory 
of linguistic behavior must posit that the determination of grammar in language acquisition involves some feature not implied by the data which the language learner meets.

Fourthly, all children in a given language community develop the same grammar, often enough in spite of radically different "inputs." It is quite likely, in fact, that in a given language community, there are children whose "inputs" are disjoint; accordingly, an adequate linguistic theory would have to show how the same gramnars can be developed from disjoint data sets. Now any such theory will have to attribute to the language acquisition capacity some feature which is not implied by the elements of the data sets.

Finally, all human languages possess the same general form of grammar; in particular all known languages have a transformational rather than a phrase-structure grammar, all have the subject-verb relation, all have quantifiers, and all have proper nouns.

I now wish to evaluate Putnam's arguments against the I.H. in light of these data, showing that (1) several of his arguments simply are not cogent; and (2) even if all Putnam's attacks on the I.H. were cogent, they are not all parts of a unified theory of linguistic behavior, and hence, at the very least, constitute a view less coherent than the I.H.

According to Putnam, many innatists cite the existence of linguistic universals as evidence in support of the I.H. But surely, he insists, there are accounts explaining the existence of linguistic universals which do not appeal to the existence of some language-specific acquisition capacity. In particular, we might imagine a community of Martians whose innate intellectual equipment is as different from ours as is compatible with their being able to have a language at all. Suppose further that Martian brains are not vastly richer than ours. Accordingly, they will find it possible to employ an infinite set of expressions only if these expressions possess a grammar consisting of a finite set of recursive rules. If the I.H. amounts to nothing more than the assertion Martians must employ a grammar, Putnan argues, then the I.H. could not possibly be false; but of course if that were so, it would not be interesting either. But in any case, we would not have to invoke the existence of a specific set of gramnars, $S$, built in the brain of both Martian and humans to account for 
the existence of linguistic universals.5

Putnam's objection, then argues that we do not have to invoke the I.H. to show that Martian and natural human languages all have some grammar or other; indeed, the existence of a grammar can be explained strictly by appealing to the need of both kinds of languages to embody a finite set of recursive rules. It must surely be conceded, whatever faults the argument may possess, that the mere existence of grammars for a set of languages does not constitute sufficient grounds for invoking the I.H. But beyond this the claim that the explanation of the existence of some grammar for each language community does not require the I.H. fails to address an important datum. In particular, an adequate theory of linguistic behavior must explain why all human languages possess the same kind of gramar. It is just this very peculiar property of all human grammars that strongly suggests the existence of a universal human grammar, deriving from a source independent of variation in human experience, name ly the innate linguistic capacities of men. Hence, that Martian has some grammar and that all human languages have some grammar, and that these two granmars differ, does not in the least impugn the claim advanced by the linguistic innatists.

Putnam offers yet another explanation for the existence of linguistic universals. Let us suppose, he says, that language-using human beings evolved independently in two or more places. Then according to the innatist hypothesis, there should be two or more types of human being descended from the two or more original populations, such that normal children of each type would fail to learn the language of the other. Since we do not ever observe such failures, and since there is only one class $S$ built into all human brains, we have to conclude, if the I.H. is true, that language-using involves a sort of evolutionary discontinuity that occurred only once. In that case, however, it is overwhelmingly likely that all human languages are descended from a single original language. But indeed, Putnan counters, the latter is likely even if the I.H. is false, since the human race is generally held to have resulted from a single evolutionary development and since then the human population, initially quite small, spread but slowly. Thus, even if language use were completely learned or invented rather than built in, it is likely that some group of humans first developed language as we know it, and then this language spread to the rest of the world. If all human languages are descended thus from a common parent, then just such highly useful features of the 
common parent such as quantifiers, proper nouns, and in general, the linguistic universals we in fact observe would be expected to survive. Thus one can explain the existence of linguistic universals merely by appealing to the "common origin" hypothesis, and hence the invocation of the I.H. is merely gratuitous.6

Whatever else may be said about this objection in particular, one must surely agree that the existence of linguistic universals does not constitute conclusive evidence for the I.H. Nevertheless, the objection overlooks a very important feature of linguistic behavior, namely, the transmission of language to children. Since children master the grammar of their language on evidence which fails to determine even a finite class of grammars, it is clear that they must supply, independently of the data, the appropriate grammar or class of grammars for their language. Thus Putnam's "common origin" hypothesis cannot supply an account of language transmission and hence is inadequate.

Many innatists claim to derive support for the I.H., Putnam asserts, from the fact that children learn languages with startling rapidity and ease. But surely, he objects, we can account for this phenomenon without appealing to the I.H. More particularly, he argues, it is not surprising that children learn a language as rapidly as they do, because they are constantly exposed to it. In comparison, language teachers claim that students can achieve mastery of a foreign language in 600 hours of direct-method instruction and achieve an incomparably greater vocabulary than that of a young child; by the age of four a child has been exposed to vastly more than 600 hours of direct-method instruction, and so has had plenty of time to acquire even a difficult language. 7

But this objection, although illuminating the fact that certain students do acquire mastery of a language in a very brief time, overlooks several important aspects of linguistic behavior. First of all, it is not so clear that leaming a second language is analogous in all relevant respects to learning a first language. One might plausibly argue that the acquisition of a second language takes the first as a model of some sort. Since the acquisition of a first language cannot bear this relation to a former language, except on pain of generating an infinite regress, it may be that the acquisition of a second language is considerably easier because of its special relation to the former. However such a suggestion might be evaluated, it is clear that the "second language" argument overlooks, like the previous 
objections, a very important aspect of linguistic behavior. It was argued above that no amount of data can explicitly determine the acquisition of even the second language. More particularly, none of the gramnatical rules found in grammars and none of the rules articulated by teachers of the language are strictly correct. Yet students who master the language do learn the exceptions to the rules. Hence the "second-language" objection just ignores that crucial feature of language learning which allows the acquisition of a grammar on the basis of degenerate evidence, and thus is inadequate to dismiss the I.H.

Putnam's final objection to the I.H. is directed against the claim that the capacity for mobilization and acquisition of language is language-specific. If we commit ourselves to the existence of a languagespecific capacity, we must analogously commit ourselves to a mathematics-specific capacity, a puzzle-solving capacity specific to certain puzzles, and so on, because these capacities, too, are not deternined completely by the data we receive. But this would be to multiply the "innate" capacities beyond the point of interest or illumination. Rather, the objection goes, it seems much more plausible to attribute the acquisition of language to general, multipurpose learning strategies which are employed in language, mathematics, puzzle-solving, and so on. 8

On close inspection, however, this objection appears to involve several difficulties. First of all, it baldly ignores the fact that nearly all children grasp the same grammar, whereas their puzzle-solving and mathematical competencies differ conspicuously. Thus the evidence for the I.H. is of an importantly different sort than that which one might raise in support of mathematics- and puzzlesolving-specific capacities. Secondly, the objection renders extrenely surprising the fact that there are linguistic universals of any sort; and in particular, it renders too surprising the fact that all children in a given language community acquire the same grammar, in spite of diverse and degenerate linguistic data.

The above criticisms shield, if correct, the I.H. from Putnam's objections. Nevertheless, I think that even if one assumes that none of these criticisms are cogent, Putnam's view still is less coherent, to say the very least, than the I.H. The I.H., that is, offers a unified, though perhaps daring explanation of the existence of linguistic universals, the anazing rapidity and thoroughness with which children achieve linguistic competence, the "creative" aspect of 
linguistic behavior, and accounts for the way in which a finite being can possess the power to recognize or potentially produce an infinite number of expressions. In contrast, these features have no comparable unity on Putnam's account. For first of all, the very reason there are linguistic universals of the sort we observe, the I.H. maintains, is just the reason why children are able to learn a grammar on the basis of degenerate evidence. On Putnam's account, in contrast, linguistic universals arose merely because language originated from a single community sharing a comnon language which contained certain linguistic features bound to be useful in any human social situation; on his view this fact has no connection wi.th the fact that children learn the language based on degenerate evidence.

Secondly, on the I.H., the conditions which permit the existence of linguistic universals insure the rapid acquisition of linguistic competence by children. Putnam, in contrast, insists that while the existence of linguistic universals is determined by social utility or the need for a recursive grammar, the rapidity of language acquisition simply comes from great exposure, and nothing more; on this view, then, the ease of language acquisition might be just as great even if there were no linguistic universals. Thirdly, on the I.H. the finite recursive nature of the grammatical rules which the language user acquires is a consequence of the nature of innate linguistic universals; on Putnan's view there is not even an accidental tie between the two.

If the choice were mine, then, I would, if Putnam's arguments are the strongest which can be brought against the I.H., cast my lot with the innatist thesis. Putnam's objections show at best, if the above criticism is just, that there will always be non-innatist interpretations of any given linguistic datum. Ihis does not surprise me too greatly: in general, evidence rarely uniquely determines theory. But I believe that no science proceeds by inventing a distinct theory for each distinct datum which seems to fall within its province, as Putnam, by example, suggests we do; rather we seek unified accounts of what we imagine, barring strong objections, to be merely diverse pieces of the same mosaic.

Indiana University, Bloomington 
NOTES

ISee in particular Nelson Goodman, "The Epistemological Argument", Synthese 17(1967).

2 Hilary Putnam, "The 'Innateness Hypothesis' and Explanatory Models in Linguistics." In Innate $\frac{\text { Ideas, }}{141}$

In particular, if the language acquisition and production faculties of the organism are such that the class of things acquired or produced can have only finite cardinality, while the class of things acquirable or producible has infinite cardinality, then the structure of language acquisition or production must in effect be characterizable by a set of recursive rules capable of generating the set of well-formed expressions of the natural language.

"More generally, it is a well-known result of elementary model theory that in any metalanguage of a language containing the first-order predicate calculus, the set-theoretic relation "E", sets, and variables ranging over sets, the cardinality of the class of extensionally distinct formation rules satis fiable by a given finite class of expressions in the object language is infinite.

$$
\begin{aligned}
& { }^{5} \text { Ibid., p. } 137 . \\
& { }^{6} \text { Ibid., p. } 140 . \\
& { }^{7} \text { Ibid., p. } 141 . \\
& { }^{8} \text { Ibid., p. } 143 .
\end{aligned}
$$

